\title{
Special issue on computational optimization and intelligence in uncertain environment
}

\author{
Jinwu Gao ${ }^{1} \cdot$ Xiaowei Chen $^{2} \cdot \mathrm{Kai} \mathrm{Yao}^{3} \cdot$ Xiangfeng Yang $^{4}$
}

Published online: 24 July 2017

(c) Springer-Verlag GmbH Germany 2017

As uncertainty is ubiquitous in the environments people reside and in various systems that protect and assist humans, the essence of intelligence in some sense lies in the agent's ability to adapt to uncertain environment and to optimize decision by efficient computation. With the rapid development of theory and methods for computational optimization in various uncertain environments, a great number of publications in this area have emerged. This special issue, focusing on the computational optimization, intelligent algorithms, and new proposals for formulations of uncertainty, provides the readers with a detailed description of the state-of-the-art theories and approaches to computational optimization and intelligence in uncertain environments. Our call for papers was warmly responded all over the world. After being rigorously referred and evaluated by the reviewers and the guest editors, only 15 papers were

Jinwu Gao

jgao@ruc.edu.cn

Xiaowei Chen

chenx@nankai.edu.cn

Kai Yao

yaokai@ucas.ac.cn

Xiangfeng Yang

yangxangfeng123@163.com

1 School of Information, Renmin University of China, Beijing 100872, China

2 School of Finance, Nankai University, Tianjin 30071, China

3 School of Management, University of Chinese Academy of Sciences, Beijing 100190, China

4 School of Information Technology and Management, University of International Business and Economics, Beijing, China accepted. The contents of these papers are briefly introduced below.

The first paper "Valuation of convertible bond under uncertain mean-reverting stock model" by Zhang et al. (2017) investigates the convertible bond of a stock which is a financial security issued by a company that gives its holder the right to exchange the bonds for the company's stock at some pre-announced ratio in the future. Based on the assumption that the stock price follows an uncertain mean-reverting differential equation, the paper derives some pricing formulas of the convertible bond and the convertible bond with callable provision. Numerical examples are given to illustrate the effectiveness of the pricing formulas.

The second paper "Time consistent fuzzy multi-period rolling portfolio optimization with adaptive risk aversion factor" by Zhou et al. (2017) investigates a time consistent multi-period rolling portfolio optimization problem under fuzzy environment. It defines an adaptive risk aversion factor to incorporate investor's changing psychological risk concerns during the intermediate periods. The future returns of risky assets are estimated by utilizing justifiable granularity principle and represented by triangular and trapezoidal fuzzy variables, respectively, and the return and risk of assets at each investment period are measured by expected value and entropy, respectively. Then the problem is formulated by a series of rolling deterministic linear programming models and solved with simplex methods, and the effectiveness of the formulation methodologies is illustrated by some numerical examples.

The third paper "The maximum flow problem of uncertain random network" by Shi et al. (2017) investigates the maximum flow problem in an uncertain random network which aims at finding the maximum amount of a flow from the source to the sink in the network. Two 
types of maximum flow models are built based on the constraints in the network, namely expected value constrained maximum flow model and chance constrained maximum flow model. These two models are transformed into the equivalent crisp models according to the operational law of uncertain random variables. Algorithms are designed based on these crisp models, and numerical experiments are performed to illustrate the algorithms.

The fourth paper "Coordinating a three level supply chain under generalized parametric interval-valued distribution of uncertain demand" by Guo et al. (2017) addresses the coordination problem for a three level supply chain in a single period model, where the uncertain demand is characterized by generalized parametric interval-valued possibility distribution. It is demonstrated that under the risk-neutral criterion, the mean supply chain profit in centralized decision is greater than the total mean supply chain profit in decentralized decision. A three level supply chain with revenue-sharing contract and return policy is studied and the analytical expressions of the optimal order quantity for different members are derived. In addition, some managerial implications in a practical supply chain coordination problem are provided.

The fifth paper "Uncertain random logic and uncertain random entailment" by Liu and Yao (2017) proposes an uncertain random logic as a generalization of probabilistic logic and uncertain logic, which can deal with complex knowledge containing random factors and human uncertainty simultaneously. A formula is derived to calculate the truth value of an uncertain random proposition. The paper also proposes an uncertain random entailment model which calculates the truth value of a function of some uncertain random propositions based on the truth values of some other functions of these uncertain random propositions. Specifically, the uncertain random modus ponens, modus tollens, and hypothetical syllogism are investigated.

The sixth paper "Stability in distribution for multifactor uncertain differential equation" by Ma et al. (2017) investigates the property of stability in distribution for the multifactor uncertain differential equations which are a type of differential equations driven multiple Liu processes. It derives a sufficient condition for a multifactor uncertain differential equation being stable in distribution, and discusses the relationship between stability in distribution, stability in mean, and stability in measure.

The seventh paper "Existence and uniqueness theorem for uncertain heat equation" by Yang and Ni (2017) verifies an existence and uniqueness theorem for the uncertain heat equations which are a type of heat equations whose heat sources are perturbed by uncertain factors. It shows the uncertain heat equation has a unique solution if its coefficients satisfy the linear growth condition and Lipstchitz condition. Specifically, some uncertain heat equations with special structures are investigated.

The eighth paper "Impact of risk levels on optimal selling to heterogeneous retailers under dual uncertainties" by Chen et al. (2017) investigates the optimal selling problem of a supplier who sells the same product to two competing retailers when the selling costs of retailers and external demand are uncertain. It is demonstrated that higher risk levels correlate with lower belief-degree costs of the two retailers and higher belief-degree sizes of the market, and a decrease in the risk borne by the channel or an increase in the competitive intensity often reduces the quantities supplied to the retailers. In addition, a contract menu of twopart tariffs with quantity controls is designed such that the optimal quantities supplied and retailer profits are the same for the relaxed and original models in the decentralized channel.

The ninth paper "Degree-constrained minimum spanning tree problem of uncertain random network" by Gao et al. (2017) investigates the degree-constrained minimum spanning tree problem in an uncertain random network which aims at finding the least weighted spanning tree subject to the constraints on node degrees of the network. An ideal chance distribution of the degree-constrained minimum spanning tree is proposed as a criterion to measure the spanning trees, and an uncertain random programming model is formulated to seek out the degree-constrained spanning tree whose chance distribution is closest to the ideal chance distribution. An algorithm is designed based on an equivalent crisp model of the formulated model, whose effectiveness is illustrated by some numerical experiments.

The tenth paper "Pure exchange competitive equilibrium under uncertainty" by Chen and Zhu (2017) considers the uncertain factors embedded in the preference of each agent, and investigates an uncertain pure exchange competitive equilibrium model. By maximizing the expected utility of each agent under the budget constraints, that problem is formulated into a quasi-variational inequality problem. Specifically, the competitive equilibrium is reached for the case with two agents and two goods.

The eleventh paper "Uncertain multi-objective optimal model of oilfield development planning and its algorithm" by $\mathrm{Ji}$ et al. (2017) takes into consideration the uncertain factors in geological, technical and economic aspects during the oilfield development, and formulates an optimization problem for the oilfield development plan by means of the uncertain multi-objective programming models. It aims to minimize the expectation of development cost and maximize the expectation of new recoverable reserves under the constraints of oil production and resources limitation. A deterministic model is obtained based on the operational law of uncertain variables, and a nondominated sorting 
genetic algorithm with elite strategy is developed to solve the model and get the Pareto solution set. In addition, the multi-attribute decision-making is also applied to select the multiple development plans.

The twelfth paper "Dual-channel supply chain competition with channel preference and sales effort under uncertain environment" by Ke and Liu (2017) investigates a dual-channel supply chain which takes into account the channel preference and sales effort to explore their effects on supply chain members' profits with uncertain information. The dual-channel supply chain in the centralized and decentralized cases are analyzed, and closed-form expressions for equilibriums in the two cases are given. It is shown the total profit of the supply chain in the centralized case is invariably higher than that in the decentralized case.

The thirteenth paper "Uncertain extensive game with application to resource allocation of national security" by Wang et al. (2017) investigates the finite extensive games with uncertain payoffs. Three different criteria are introduced to describe players behaviors in different decisionmaking situations, which are the expected value criterion, the optimistic value criterion, and the pessimistic value criterion. Under each criterion, the concepts of uncertain equilibrium and uncertain subgame perfect equilibrium are proposed, and theorems are given to the existence of these two equilibria. As an application, the resource allocation problem for national security is formulated and analyzed using the uncertain extensive game, and the results show that different decision criteria may lead to different subgame perfect equilibria.

The fourteenth paper "Optimization of water allocation decisions under uncertainty: the case of option contracts" by Ha and Gao (2017) introduces an exact two-stage mixedinteger programming model to allocate the water resource under uncertainty. It presents a case study on the water allocation with option contracts, and analyzes the factors influencing the willingness of signing the option contracts. It is shown that the water option contract can help mitigate the water shortage and increase economic benefits.

The last paper "Optimizing influence diffusion in a social network with fuzzy costs for targeting nodes" by $\mathrm{Ni}$ et al. (2017) focuses on the problem of optimizing the complete influence time in a social network where the cost for targeting each individual is characterized by fuzzy variables. Based on different decision criteria, three models are proposed to describe the problem, which are the expected value model, the chance-constrained programming model, and the dependence-chance programming model. By integrating the fuzzy simulation with a modified greedy algorithm, a hybrid intelligence algorithm is designed to solve the models, and its effectiveness is shown by the numerical experiments.
As guest editors of this special issue, we would like to express our appreciation to all the authors for their contributions and to the reviewers for their valuable comments. We would also like to express our thanks to Professor Vincenzo Loia for providing us the opportunity to organize this special issue as well as for his introductions in the editorial process.

\section{References}

Chen HR, Wang XB, Liu ZB, Zhao RQ (2017) Impact of risk levels on optimal selling to heterogeneous retailers under dual uncertainties. J Ambient Intell Hum Comput. doi:10.1007/ s12652-017-0481-9

Chen QQ, Zhu YG (2017) Pure exchange competitive equilibrium under uncertainty. J Ambient Intell Hum Comput. doi:10.1007/ s12652-017-0500-x

Gao X, Jia LF, Kar S (2017) Degree-constrained minimum spanning tree problem of uncertain random network. J Ambient Intell Hum Comput. doi:10.1007/s12652-017-0493-5

Guo ZZ, Liu YK, Liu Y (2017) Coordinating a three level supply chain under generalized parametric interval-valued distribution of uncertain demand. J Ambient Intell Hum Comput. doi:10.1007/s12652-017-0472-x

Ha MH, Gao ZC (2017) Optimization of water allocation decisions under uncertainty: the case of option contracts. J Ambient Intell Hum Comput. doi:10.1007/s12652-017-0551-Z

Ji XY, Yan S, Feng SY (2017) Uncertain multi-objective optimal model of oilfield development planning and its algorithm. J Ambient Intell Hum Comput. doi:10.1007/s12652-017-0530-4

Ke H, Liu JJ (2017) Dual-channel supply chain competition with channel preference and sales effort under uncertain environment. J Ambient Intell Hum Comput. doi:10.1007/s12652-017-0502-8

Liu YH, Yao K (2017) Uncertain random logic and uncertain random entailment. J Ambient Intell Hum Comput. doi:10.1007/ s12652-017-0465-9

Ma WM, Liu LY, Gao R, Zhang XL, Zhang XF (2017) Stability in distribution for multifactor uncertain differential equation. J Ambient Intell Hum Comput. doi:10.1007/s12652-017-0517-1

Ni YD, Shi QN, Wei ZY (2017) Optimizing influence diffusion in a social network with fuzzy costs for targeting nodes. J Ambient Intell Hum Comput. doi:10.1007/s12652-017-0552-y

Shi G, Sheng YH, Ralescu DA (2017) The maximum flow problem of uncertain random network. J Ambient Intell Hum Comput. doi:10.1007/s12652-017-0495-3

Wang Y, Luo S, Gao JW (2017) Uncertain extensive game with application to resource allocation of national security. J Ambient Intell Hum Comput. doi:10.1007/s12652-017-0538-9

Yang XF, Ni YD (2017) Existence and uniqueness theorem for uncertain heat equation. J Ambient Intell Hum Comput. doi:10.1007/ s12652-017-0479-3

Zhang ZQ, Liu WQ, Zhang XD (2017) Valuation of convertible bond under uncertain mean-reverting stock model. J Ambient Intell Hum Comput. doi:10.1007/s12652-017-0487-3

Zhou JD, Li X, Kar S, Zhang GQ, Yu HT (2017) Time consistent fuzzy multi-period rolling portfolio optimization with adaptive risk aversion factor. J Ambient Intell Hum Comput. doi:10.1007/ s12652-017-0478-4 